\title{
Quantification of the interfacial and bulk contributions to the longitudinal spin Seebeck effect
}

\author{
P. Jiménez-Cavero, ${ }^{1,2}$ I. Lucas, ${ }^{1,2, a)}$ D. Bugallo, ${ }^{3}$ C. López-Bueno, ${ }^{3}$ R. Ramos, ${ }^{3}$ P. A. Algarabel,,${ }^{2,1}$ M. R. \\ Ibarra, ${ }^{1,2,4} \mathrm{~F}$. Rivadulla, ${ }^{3}$ and L. Morellón ${ }^{1,2}$ \\ ${ }^{1)}$ Instituto de Nanociencia y Materiales de Aragón, Universidad de Zaragoza-CSIC, 50018 Zaragoza, \\ Spain \\ ${ }^{2)}$ Departamento de Física de la Materia Condensada, Universidad de Zaragoza, 50009 Zaragoza, \\ Spain \\ ${ }^{3)}$ Centro de Investigación en Química Biolóxica e Materiais Moleculares (CIQUS), Universidade de Santiago de Compostela, \\ 15782 Santiago de Compostela, Spain \\ 4) Laboratorio de Microscopías Avanzadas, Universidad de Zaragoza, 50018 Zaragoza, \\ Spain
}

(Dated: 12 February 2021)

We report the disentanglement of bulk and interfacial contributions to the thermally excited magnon spin current in the spin Seebeck effect under static heating. For this purpose, we have studied the dependence of the inverse Spin Hall voltage and the thermal conductivity on the magnetic layer thickness. Knowledge of these quantities allows us to take into account the influence of both sources of thermal spin current in the analysis of the voltage dependence. The magnetic layer thickness modulates the relative magnitude of the involved thermal drops for a fixed total thermal difference throughout the sample. In the end, we attain the separate contributions of both sources of thermal spin current - bulk and interfacial — and obtain the value of the thermal magnon accumulation length scale in maghemite, which we find to be 29(1) nm. According to our results, bulk magnon accumulation dominates the spin Seebeck effect in our studied range of thicknesses, but the interfacial component is by no means negligible.

PACS numbers: 72.15.Jf, 72.25.b, 81.15.-Z

The spin Seebeck effect (SSE) is a complex phenomenon that lies at the intersection between several spintronics subfields. It is defined as the generation of a spin current in a magnetic material (FM) subjected to a thermal gradient, standing as one of the major spin-caloritronics topics ${ }^{1}$. However, the detection of the SSE makes use of tools from spinorbitronics, since it is commonly achieved by spin current to charge current conversion by means of the inverse spin Hall effect (ISHE) observed in a nonmagnetic heavy metal (NM) adjacent to the magnetic material $(\mathrm{FM})^{2}$. Furthermore, these thermal spin currents have been proved to be of magnonic origin, which means that magnonics are also involved in $\mathrm{SSE}^{3,4}$. This complexity affects both theoretical and experimental aspects and is evidenced, e.g., by the difficulties found in the definition of a standard SSE coefficient ${ }^{5,6}$.

Part of this intricacy comes from the fact that there are different physical mechanisms contributing to the $\mathrm{SSE}^{7}$. Two main theories have been developed so far which describe two different sources for a magnon spin current in a FM/NM system subjected to a thermal gradient. The first one points to the temperature difference between the metal electrons and the magnetic magnons at the interface, such that the excited spin current is given by ${ }^{4,8}$ :

$$
J_{\mathrm{S}}^{\mathrm{i}} \propto \Delta T_{\mathrm{i}}^{\mathrm{NM} / \mathrm{FM}},
$$

where $\Delta T_{\mathrm{i}}^{\mathrm{NM} / \mathrm{FM}}$ denotes the interfacial thermal drop.

The second origin of the thermal spin current lies on the thermal gradient present in the bulk of the FM layer itself,

\footnotetext{
a)ilucas@unizar.es
}

rather than the temperature difference at the interface $e^{9-12}$. This gradient creates a magnon accumulation which acts as a spin potential for the spin current. In this case, the spin current is determined by a finite magnon propagation length scale $\left(\Lambda_{\mathrm{m}}\right)$ and follows the expression ${ }^{10,12}$ :

$$
J_{\mathrm{s}}^{\mathrm{b}} \propto \frac{\cosh \left(t_{\mathrm{FM}} / \Lambda_{\mathrm{m}}\right)-1}{\sinh \left(t_{\mathrm{FM}} / \Lambda_{\mathrm{m}}\right)} \nabla T_{\mathrm{FM}},
$$

where $\nabla T_{\mathrm{FM}}$ is the temperature gradient across the $\mathrm{FM}$ layer and $t_{\mathrm{FM}}$ denotes its thickness.

We empashize that both mechanisms arise in the presence of a thermal gradient and thus meet the definition of the SSE, but they actually represent different physical sources for thermally excited magnon spin currents.

Nowadays the logitudinal spin Seebeck effect (LSSE) is widely used because of experimental simplicity, and most theoretical efforts have focused on it $^{13}$. In the LSSE an out-ofplane thermal gradient is applied perpendicularly to a magnetic field. The excited spin current is parallel to the thermal gradient and according to the ISHE phenomenology, the generated voltage can be measured in the transverse direction (perpendicular to both thermal gradient and magnetic field).

Whilst time-resolved optical approaches of SSE measurements have been able to selectively excite only interfacial LSSE ( $i$ LSSE) ${ }^{14,15}$ and the $b$ LSSE has been unambiguously detected using a magnon-valve structure ${ }^{16}$, the relative contributions in a standard DC measurement in which the whole sample is subjected to a thermal gradient have not been addressed. In these conditions, both thermal spin current sources coexist and contribute to the signal. Notwithstanding, in most of previous works one of the existing models is chosen, as- 
suming that only one of the mechanisms is present or relevant. Therefore, a relative quantification of both contributions is still lacking. This is however an important issue, required for a deeper understanding of the LSSE. In addition, it is also desirable from a practical point of view as it will give hints concerning the design of materials and devices to be used in potential applications.

In this work, we disentangle the contributions of $i \mathrm{LSSE}$ and $b$ LSSE (bulk LSSE) within a static SSE experiment. We make use of following definitions for the corresponding coefficients $\left(S^{\mathrm{i}}\right.$ and $\left.S^{\mathrm{b}}\right)$ :

$$
S^{\mathrm{i}}=\frac{\Delta V_{\mathrm{ISHE}}^{i}}{d_{y} \Delta T^{\mathrm{NM} / \mathrm{FM}}} \quad \text { and } \quad S^{\mathrm{b}}=\frac{\Delta V_{\mathrm{ISHE}}^{b}}{d_{y} \Delta T_{\mathrm{FM}}},
$$

where $d_{y}$ is the distance between the transverse contacts to measure the transverse ISHE voltage $\left(\Delta V_{\text {ISHE }}\right)$. These definitions make use of the actual temperature difference driving each effect, rather than the overall temperature difference. This normalization is favored by the SSE measurement method known as heat flux method ${ }^{6,17,18}$, which measures the heat current flowing through the sample instead of the total temperature difference (used in the temperature difference method).

Our approach to the quantification of $i \mathrm{LSSE}$ and $b \mathrm{LSSE}$ contributions is based on the study of the LSSE as a function of $t_{\mathrm{FM}}$. Under a fixed total thermal difference across the whole sample $\Delta T$ (typical experimental condition in LSSE static experiments), the thermal drops across the FM layer thickness and at the NM/FM interface change upon changing $t_{\mathrm{FM}}$. Consequently, $J_{\mathrm{s}}^{b}$ and $J_{\mathrm{s}}^{i}$ change accordingly, since they are driven by those thermal differences (recall Eqs. 1 and 2). However, this issue has been omitted in previous studies on the $t_{\mathrm{FM}}$ dependence of LSSE employing the temperature difference approach, although it represents a source of modulation of the contribution of both LSSE mechanisms. Typically, only the dependence related to the $\Lambda_{\mathrm{m}}$ (prefactor in equation 2) is addressed, whereas the influence of $t_{\mathrm{FM}}$ in the temperature drop in FM, $\Delta T_{\mathrm{FM}}$, has not been considered so far. To evaluate this, knowledge on the cross-plane thermal conductivities is needed to quantify the gradients. Therefore, a key point in our work is the assessment of the thermal conductivities for thin films of different thicknesses. An alternative to circumvent the thickness variation of $\Delta T_{\mathrm{FM}}$ is offered by the heat flux method ${ }^{19}$. However, to separate the contributions to the LSSE, knowledge of the thermal conductivities is necessary.

In sum, in a typical experiment of LSSE as a function of $t_{\mathrm{FM}}$ in which $\Delta T$ is fixed to the same value for all $t_{\mathrm{FM}}$, we consider the modulation of the thermally excited spin current $J_{\mathrm{S}}$ by three different means: (1) the existence of a magnon propagation length scale $\Lambda_{\mathrm{m}}$ comparable to the dimensions of our samples, (2) the change of the involved thermal drops due to the variation of the FM layer thickness in relation to the rest of the stacked layers, and (3) the dependence of the thermal conductivity of the FM layer on its thickness.

The studied samples consisted of epitaxial thin films of insulating ferrimagnetic $\gamma-\mathrm{Fe}_{2} \mathrm{O}_{3}$ (maghemite) deposited on (001) oriented $\mathrm{MgO}$ substrates of $0.5 \mathrm{~mm}$ of thickness, and an additional layer of Pt on top of maghemite for spin-to-charge

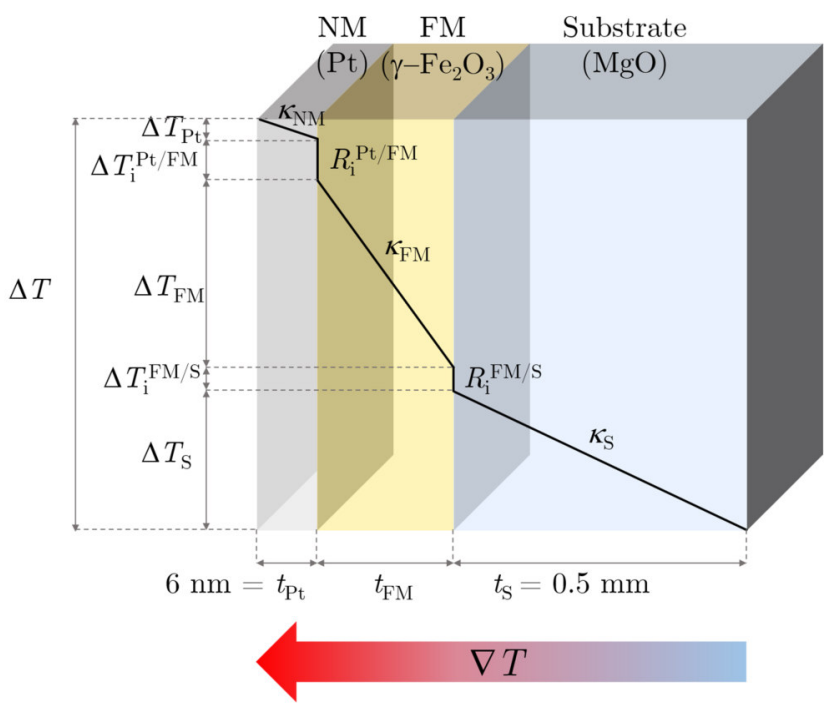

FIG. 1. Depiction of the thermal differences established in the LSSE experiment through the studied sample, as expressed in Eq. 4. The graded arrow shows the direction of the thermal gradient.

current conversion. The thickness of the Pt layer is held at 6 $\mathrm{nm}$ through all studied samples. Maghemite is an ideal material to study LSSE because of its insulating behavior ruling Anomalous Nernst Effect contribution to the voltage ${ }^{20-22}$. However, proximity magnetism induced in the Pt layer by interdiffusion of $\mathrm{Fe}$ may originate an ANE contribution. To avoid this effect, Pt was deposited at room temperature ${ }^{23,24}$. Details about the fabrication of the samples can be found elsewhere, along with the description of the experimental setup for the LSSE measurements ${ }^{25}$. Moreover, the quality of interfaces affects the magnitude of the $\mathrm{LSSE}^{26}$; therefore, we assessed the interfacial roughness by X-ray reflectivity, finding comparable values $<1 \mathrm{~nm}$ for every $t_{\mathrm{FM}}$ (supplementary material).

In our setup the temperature difference between the hot and cold baths (i.e. the temperature drop $\Delta T$ across the whole sample including $\mathrm{MgO}$ substrate) is controlled and measured. The temperature profile will show two main features: (1) a change in its slope from layer to layer because of the different thermal conductivities and (2) a discontinuity at the interfaces due to the interfacial thermal resistance, also known as Kapitza resistance. Therefore, as illustrated in Fig. 1, we can split the total temperature difference into thermal drops along the sample:

$$
\Delta T=\Delta T_{\mathrm{Pt}}+\Delta T_{\mathrm{i}}^{\mathrm{Pt} / \mathrm{FM}}+\Delta T_{\mathrm{FM}}+\Delta T_{\mathrm{i}}^{\mathrm{FM} / \mathrm{S}}+\Delta T_{\mathrm{S}}
$$

which accounts for the temperature drop in the Pt layer, at the Pt/FM interface, in the FM layer, at the FM/substrate interface, and across the substrate. In this equation, the thermal drops originated by the thermal contacts of the sample with the baths ${ }^{27}$ are disregarded, thus the values obtained in the determination of $S^{\mathrm{i}}$ and $S^{\mathrm{b}}$ are underestimated. However, the quantification of the relative contributions to $\Delta V_{\text {ISHE }}$ should be unaffected by this systematic error. The heat flux 


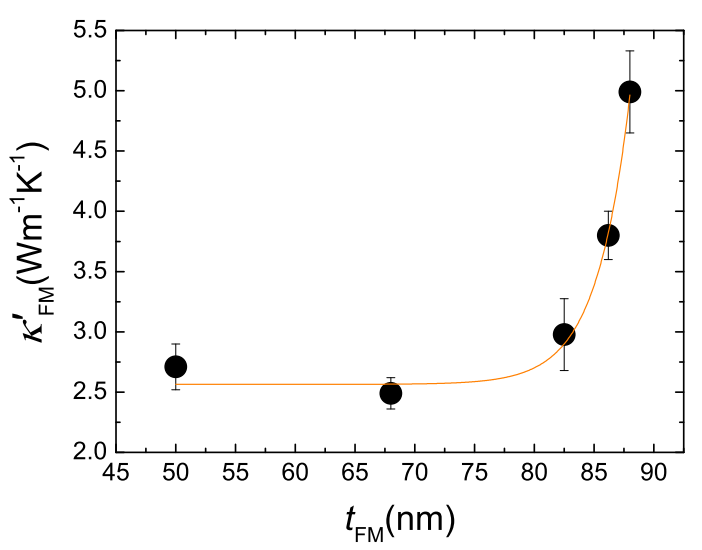

FIG. 2. Effective thermal conductivity of maghemite epitaxial thin films as a function of the thickness. Solid line represents an experimental fit to a sigmoid function used for interpolation of $\kappa_{\mathrm{FM}}^{\prime}$ at the desired $t_{\mathrm{FM}}$.

$\operatorname{method}^{6,17,18}$ offers another possibility to circumvent this error.

According to a simple thermal model the heat flux is constant through the whole sample:

$$
\begin{gathered}
J_{\mathrm{Q}}=\left(\frac{t_{\mathrm{Pt}}}{\kappa_{\mathrm{Pt}}}+\frac{t_{\mathrm{FM}}}{\kappa_{\mathrm{FM}}}+\frac{t_{\mathrm{S}}}{\kappa_{\mathrm{S}}}+R_{\mathrm{i}}^{\mathrm{Pt} / \mathrm{FM}}+R_{\mathrm{i}}^{\mathrm{FM} / \mathrm{S}}\right)^{-1} \Delta T= \\
=\frac{1}{R_{\mathrm{T}}} \Delta T=\frac{\kappa_{\mathrm{FM}}}{t_{\mathrm{FM}}} \Delta T_{\mathrm{FM}}=\frac{1}{R_{\mathrm{i}}^{\mathrm{P} / / \mathrm{FM}}} \Delta T_{\mathrm{i}}^{\mathrm{Pt} / \mathrm{FM}}
\end{gathered}
$$

$t$ 's denote thicknesses and $\kappa$ 's denote thermal conductivities; $R_{\mathrm{i}}^{\mathrm{Pt} / \mathrm{FM}}\left(R_{\mathrm{i}}^{\mathrm{FM} / \mathrm{S}}\right)$ is the thermal resistance coming from the $\mathrm{Pt} / \mathrm{FM}$ (FM/substrate) interface; and $R_{\mathrm{T}}$ represents the thermal resistance of the system as a whole, i.e., the composition of the thermal resistances of each layer and interface.

As pointed above, the first step is establishing the actual thermal drop across the magnetic layer as well as through the FM/Pt interface. This is especially troublesome when dealing with thin films in which thermal transport properties differ from those of the bulk materials and are not handily characterizable $^{28}$. However, the knowledge of these quantities is paramount for the quantitative analysis of the LSSE. This means that measurement of the thermal conductivity of the thin film is mandatory, since its value must be included into even the simplest thermal model along with that of the substrate.

In this work, the cross-plane thermal conductivity of maghemite thin films as well as $\mathrm{MgO}$ substrate were determined by the $3 \omega$ method $^{29}$ (supplementary material). It is important to note that the determined thin film conductivity is not the intrinsic $\kappa_{\mathrm{FM}}$ but an effective $\kappa_{\mathrm{FM}}^{\prime}$ which also accounts for the thermal loss at the $\gamma-\mathrm{Fe}_{2} \mathrm{O}_{3} / \mathrm{MgO}$ interface $R_{\mathrm{i}}^{\mathrm{FM} / \mathrm{S} 30}$.

$$
\kappa_{\mathrm{FM}}^{\prime}=\frac{\kappa_{\mathrm{FM}}}{1+R_{\mathrm{i}}^{\mathrm{FM} / \mathrm{S}} \kappa_{\mathrm{FM}} / t_{\mathrm{FM}}}
$$

We will use this effective $\kappa_{\mathrm{FM}}^{\prime}$ to calculate $\Delta T_{\mathrm{FM}}$. This means that hereafter, $\Delta T_{\mathrm{i}}^{\mathrm{FM} / \mathrm{S}}$ will be contained in $\Delta T_{\mathrm{FM}}$.
We measure $\kappa_{\mathrm{FM}}^{\prime}$ at $T=300 \mathrm{~K}$ for different $t_{\mathrm{FM}}$ up to the thickness range of the samples we studied in the LSSE experiments. The thereby obtained values of the thermal conductivities at $300 \mathrm{~K}$ are depicted in Fig. 2. They follow a rather complex dependence, which may be attributed to the presence of defects rapidly changing with the film thickness; a possible candidate are the antiphase boundaries (APBs) which are usually shown by spinel structures (such as maghemite) and whose density decrease on increasing thickness ${ }^{31}$. A detailed analysis of this dependence is however beyond the scope of this work. From this experimental curve, we interpolate the values of $\kappa_{\mathrm{FM}}^{\prime}$ at the $t_{\mathrm{FM}}$ of the samples studied in the LSSE experiments. Concerning $\mathrm{MgO}$ substrate, we measured $\kappa_{\mathrm{S}}=44.2(5) \mathrm{W} \mathrm{m}^{-1} \mathrm{~K}^{-1}$ at $T=300 \mathrm{~K}$, in agreement with reported values for $\mathrm{MgO}$ single crystals $^{32,33}$.

The thermal resistance of the Pt layer is lower than the rest of the terms by two orders of magnitude, due to its small thickness $t_{\mathrm{Pt}}=6 \mathrm{~nm}$ and large thermal conductivity $\kappa_{\mathrm{Pt}}=64$ $\mathrm{Wm}^{-1} \mathrm{~K}^{-134}$. The thermal resistances of the stacked layers are $R_{\mathrm{MgO}} \sim 10^{-5} \mathrm{~W}^{-1} \mathrm{~m}^{2} \mathrm{~K}, R_{\mathrm{FM}}$ ranges from $\sim 10^{-9}$ to $10^{-8}$ $\mathrm{W}^{-1} \mathrm{~m}^{2} \mathrm{~K}$, and from literature $R_{\mathrm{i}} \sim 10^{-9} \mathrm{~W}^{-1} \mathrm{~m}^{2} \mathrm{~K}^{34,35}$; all of them exceed $R_{\mathrm{Pt}} \sim 10^{-11} \mathrm{~W}^{-1} \mathrm{~m}^{2} \mathrm{~K}$ by at least two orders of magnitude. This means that we can hence neglect the temperature drop in the Pt layer in Eq. 4 and its resistance contribution to Eq. 5.

Eq. 5 allows us to write every temperature drop in terms of the known $\Delta T$ :

$$
\begin{aligned}
\Delta T_{\mathrm{FM}} & \approx \frac{\kappa_{\mathrm{S}} t_{\mathrm{FM}}}{\kappa_{\mathrm{S}} t_{\mathrm{FM}}+\kappa_{\mathrm{FM}}^{\prime} t_{\mathrm{S}}} \Delta T \\
\Delta T_{\mathrm{i}}^{\mathrm{Pt} / \mathrm{FM}} & \approx \frac{\kappa_{\mathrm{S}} \kappa_{\mathrm{FM}}^{\prime} R_{\mathrm{i}}^{\mathrm{P} / \mathrm{FM}}}{\kappa_{\mathrm{S}} t_{\mathrm{FM}}+\kappa_{\mathrm{FM}}^{\prime} t_{\mathrm{S}}} \Delta T
\end{aligned}
$$

where we have taken a second approximation: $\kappa_{\mathrm{S}} \kappa_{\mathrm{FM}} R_{\mathrm{i}}^{\mathrm{Pt} / \mathrm{FM}} \ll \kappa_{\mathrm{FM}} t_{\mathrm{S}}$, which is reasonable, given the substrate thickness as well as the typical values of $R_{\mathrm{i}}$.

Once the thermal conductivities $\kappa_{\mathrm{FM}}^{\prime}$ and $\kappa_{\mathrm{S}}$ have been estimated in this way, we proceed to the LSSE experiments for different $t_{\mathrm{FM}}$. It has been shown that variations in the thermal contacts between the sample and the baths are source of error $^{17,27}$; care was taken to minimize these errors by ensuring that the same heat power sustained a similar $\Delta T$ for all samples. Alternatively, other approaches measure the heat flux instead $^{6,17,36}$.

In Fig. 3 the measured dependence of transverse voltage $\Delta V_{\text {ISHE }}$ on $t_{\mathrm{FM}}$ is shown. Following a widespread practice, these quantities have been normalized by the total thermal difference $\Delta T^{13,37}$, using the slopes of the linear fits of $\Delta V_{\text {ISHE }}$ as a function of different $\Delta T$ (see the inset of Fig. 3).

However, this means that, according to Eq. 7, $\Delta T_{\mathrm{FM}}$ and $\Delta T_{\mathrm{i}}$ depend on $t_{\mathrm{FM}}$ in Fig. 3. Hence, the observed behavior comprises not only the effect of $\Lambda_{\mathrm{m}}$ (as assumed by previous works) but also the variation of the thermal differences driving the LSSE (bulk and interfacial). To rigorously take into account both effects, the scaling with the corresponding thermal drops should be used instead ${ }^{17,18}$, as proposed in Eq. 3 . For this, we rewrite the spin Seebeck coefficients as a function 
of the measured total $\Delta T$, using Eq. 7:

$$
\begin{aligned}
& \Delta V_{\mathrm{ISHE}}^{\mathrm{i}} \approx S^{\mathrm{i}} \cdot d_{y} \cdot \frac{\kappa_{\mathrm{S}} \kappa_{\mathrm{FM}}^{\prime} R_{\mathrm{i}}^{\mathrm{Pt} / \mathrm{FM}}}{\kappa_{\mathrm{S}} t_{\mathrm{FM}}+\kappa_{\mathrm{FM}}^{\prime} t_{\mathrm{S}}} \Delta T \\
& \Delta V_{\mathrm{ISHE}}^{\mathrm{b}} \approx S^{\mathrm{b}} \cdot d_{y} \frac{\kappa_{\mathrm{S}} t_{\mathrm{FM}}}{\kappa_{\mathrm{S}} t_{\mathrm{FM}}+\kappa_{\mathrm{FM}}^{\prime} t_{\mathrm{S}}} \Delta T
\end{aligned}
$$

According to ISHE phenomenology, ISHE transverse voltage $\Delta V_{\mathrm{ISHE}}$ and the exciting spin current density $J_{\mathrm{S}}$ are related by $\Delta V_{\mathrm{ISHE}} \propto \theta_{\mathrm{SH}} \rho_{\mathrm{c}} J_{\mathrm{S}}{ }^{38}$, where $\theta_{\mathrm{SH}}$ is the so-called spin Hall angle accounting for the efficiency of the spin-to-charge conversion, and $\rho_{\mathrm{c}}$ is the longitudinal electrical resistivity of the NM ISHE medium (here, Pt). Depending on the mechanism governing ISHE, $\theta_{\mathrm{SH}}$ may depend on $\rho_{\mathrm{c}}{ }^{39-41}$. However, in this experiment, Pt layer thickness is the same for all samples, resulting in constant $\rho_{\mathrm{c}}$. Therefore, $\Delta V_{\mathrm{ISHE}} \propto J_{\mathrm{s}}$. In sight of this fact and Eqs. 1, 2 and 8, we note that, unlike $S^{\mathrm{i}}, S^{\mathrm{b}}$ depends on $t_{\mathrm{FM}}$ and can be expressed as:

$$
S^{\mathrm{b}}=A \cdot \frac{1}{t_{\mathrm{FM}}} \frac{\cosh \left(t_{\mathrm{FM}} / \Lambda_{\mathrm{m}}\right)-1}{\sinh \left(t_{\mathrm{FM}} / \Lambda_{\mathrm{m}}\right)}
$$

where the coefficient $A$ describes the thickness-independent term.

Altogether, the measured transverse voltage $\Delta V_{\text {ISHE }}$ will be

$$
\begin{aligned}
\Delta V_{\mathrm{ISHE}}=\Delta V_{\mathrm{ISHE}}^{\mathrm{i}}+\Delta V_{\mathrm{ISHE}}^{\mathrm{b}}=S^{\mathrm{i}} \cdot d_{y} \cdot \Delta T_{\mathrm{i}}^{\mathrm{Pt} / \mathrm{FM}} & +S^{\mathrm{b}} \cdot d_{y} \cdot \Delta T_{\mathrm{FM}} \approx \\
& \approx\left(S^{\mathrm{i}} \cdot d_{y} \cdot \frac{\kappa_{\mathrm{S}} \kappa_{\mathrm{FM}}^{\prime} R_{\mathrm{i}}^{\mathrm{Pt} / \mathrm{FM}}}{\kappa_{\mathrm{S}} t_{\mathrm{FM}}+\kappa_{\mathrm{FM}}^{\prime} t_{\mathrm{S}}}+A \cdot d_{y} \cdot \frac{\cosh \left(t_{\mathrm{FM}} / \Lambda_{\mathrm{m}}\right)-1}{\sinh \left(t_{\mathrm{FM}} / \Lambda_{\mathrm{m}}\right)} \frac{\kappa_{\mathrm{S}}}{\kappa_{\mathrm{S}} t_{\mathrm{FM}}+\kappa_{\mathrm{FM}}^{\prime} t_{\mathrm{S}}}\right) \Delta T
\end{aligned}
$$

A fit of this expression to the experimental data, shown in Fig. 3, provides (supplementary material) $\Lambda_{\mathrm{m}}=29(1)$ $\mathrm{nm}, S^{\mathrm{i}} R_{\mathrm{i}}^{\mathrm{Pt} / \mathrm{FM}}=1.83(6) \times 10^{-9}\left(\mathrm{Vm}^{-1} \mathrm{~K}^{-1}\right) \cdot\left(\mathrm{W}^{-1} \mathrm{~m}^{2} \mathrm{~K}\right)$ and $A=40.8(9) \times 10^{-9} \mathrm{~V} \cdot \mathrm{K}^{-1}$. Given the reported order of magnitude of interfacial thermal resistances $R_{\mathrm{i}} \sim 10^{-9}-10^{-8}$ $\mathrm{W}^{-1} \mathrm{~m}^{2} \mathrm{~K}$, we can also establish that $S^{\mathrm{i}}$ order of magnitude must lie between 0.1 and $1 \mathrm{Vm}^{-1} \mathrm{~K}^{-1}$, in agreement with previous estimations in other systems $7,15,26$. Concerning $S^{\mathrm{b}}$, the value of $A$ provides from $S^{\mathrm{b}}=0.65(6) \mathrm{Vm}^{-1} \mathrm{~K}^{-1}$ for the thinnest sample down to $S^{\mathrm{b}}=0.46(4) \mathrm{Vm}^{-1} \mathrm{~K}^{-1}$ for the thickest one.

We can now compute the relative contribution of each source $-b$ LSSE and $i$ LSSE - to the total $\Delta V_{\text {ISHE }}$ output in our $t_{\mathrm{FM}}$ range for an experiment in which a total $\Delta T$ is estab-

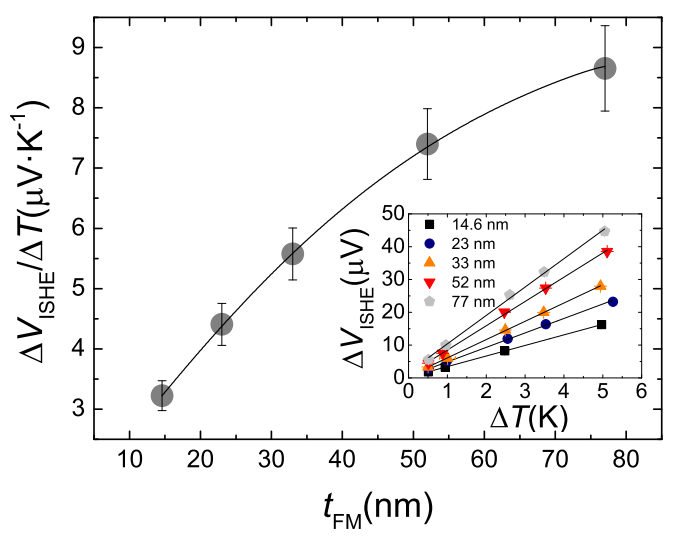

FIG. 3. Transverse voltage $\Delta V_{\text {ISHE }} / \Delta T$ measured for every different $t_{\mathrm{FM}}$ and fit of Eq. 10 to the experimental data. Inset: linear fits of $\Delta V_{\text {ISHE }}$ as a function of $\Delta T$. lished. For this, we calculate $\Delta V_{\text {ISHE }}^{\mathrm{i}}$ and $\Delta V_{\text {ISHE }}^{\mathrm{b}}$ generated per Kelvin of total thermal drop $\Delta T$ from Eqs. 3 and 7 (i.e., the two addends between brackets in Eq. 10). The result is shown in Fig. 4.As one could expect, the influence of $R_{\mathrm{i}}^{\mathrm{Pt} / \mathrm{FM}}$ is greater for thinner samples and consequently $i$ LSSE contribution to the overall signal reduces as $t_{\mathrm{FM}}$ is increased, and reversely for $b \mathrm{LSSE}$.

In summary, we have experimentally separated the bulk magnon accumulation and purely interfacial contributions to the LSSE in static heating conditions. We have done so by studying the LSSE as a function of the FM layer thickness in $\gamma-\mathrm{Fe}_{2} \mathrm{O}_{3} / \mathrm{Pt}$ bilayers, and taking into account the relative change of the present thermal drops along the sample on vary-

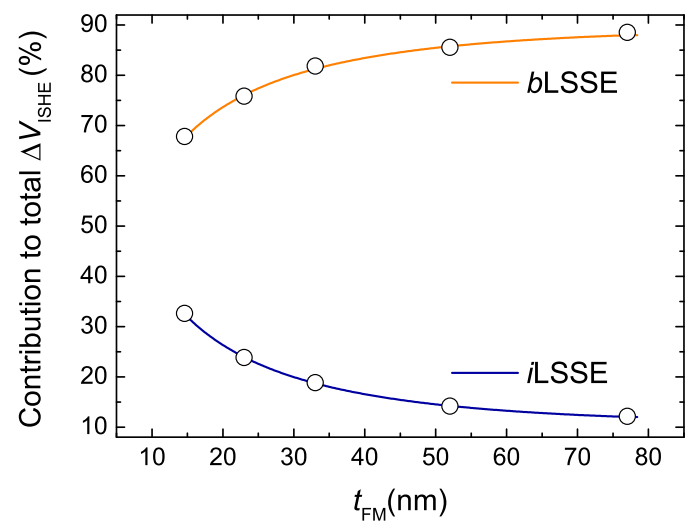

FIG. 4. Relative contribution $\Delta V_{\text {ISHE }}^{\mathrm{i}}$ and $\Delta V_{\text {ISHE }}^{\mathrm{b}}$ to the total $\Delta V_{\text {ISHE}}$. Note that these percentages cannot be directly extended to $S^{\mathrm{i}}$ and $S^{\mathrm{b}}$, given that they do not share the voltage dependences on $t_{\mathrm{FM}}$ (see Eq. 8). 
ing the FM thickness. To that end, we have also measured the thickness dependence of the thermal conductivity of $\gamma-\mathrm{Fe}_{2} \mathrm{O}_{3}$ thin films by means of the $3 \omega$ method. With this, we managed to fit the addition of theoretical models for both sources to our experimental data. This allowed us to in the end compute the separate contribution of each source of thermal spin current. We found that although bulk component dominates in the range of thickness of our samples, the interfacial contribution is not negligible against it, as it represents from $\approx 33 \%$ to $\approx 12 \%$ of the total voltage. Besides, $b$ LSSE coefficient values were determined: it varies between $S^{\mathrm{b}}=0.68(6) \mathrm{Vm}^{-1} \mathrm{~K}^{-1}$ for the thinnest sample $\left(t_{\mathrm{FM}}=14.5 \mathrm{~nm}\right)$ and $S^{\mathrm{b}}=0.46(4)$ $\mathrm{Vm}^{-1} \mathrm{~K}^{-1}$ for the thickest one $\left(t_{\mathrm{FM}}=77 \mathrm{~nm}\right)$. $i$ LSSE coefficient order of magnitude was estimated to lie between $S^{\mathrm{i}} \sim 0.1$ and $1 \mathrm{Vm}^{-1} \mathrm{~K}^{-1}$, in agreement with the values reported in literature for other systems $s^{7,15,26}$. The fit also provided a thermal magnon accumulation length in $\gamma-\mathrm{Fe}_{2} \mathrm{O}_{3}$ of $\Lambda_{\mathrm{m}}=29(1)$ $\mathrm{nm}$. In literature, $\Lambda_{\mathrm{m}}$ at room temperature corresponding to other ferrimganetic oxides can be found: $\Lambda_{\mathrm{m}}=17 \mathrm{~nm}$ for halfmetal $\mathrm{Fe}_{3} \mathrm{O}_{4}{ }^{42}$ or $\Lambda_{\mathrm{m}} \sim 100 \mathrm{~nm}$ for insulating $\mathrm{YIG}^{26}$ were reported using the temperature difference method. However, one must be cautious before comparing our result, because those works only considered the influence of the thermally excited magnon propagation length on the thickness dependence of LSSE. More recent works using different approaches to investigate this dependence circumvent the change of $\Delta T_{\mathrm{FM}}$. For example, Noack et al. studied the LSSE time evolution in YIG thin films heating with microwave pulses, finding $\Lambda_{\mathrm{m}} \sim 425$ $\mathrm{nm}^{43}$; and Venkat et al., using the heat flux method, reported $\Lambda_{\mathrm{m}}=19(2) \mathrm{nm}$ for $\mathrm{Fe}_{3} \mathrm{O}_{4}$ thin films ${ }^{19}$. Still, only the bulk effect is considered to explain the results.

These results reassure maghemite's potential in spincaloritronic devices and bring insight into the global picture of the LSSE. In particular, the relative quantification of bulk and interfacial contributions improves the precision of its description, therefore assisting a deeper understanding of the effect.

\section{SUPPLEMENTARY MATERIAL}

See supplementary material for details of the $3 \omega$ method, the structural characterization of samples, and the fit parameters initialization.

\section{ACKNOWLEDGMENTS}

This work was supported by the Spanish Ministry of Science (through Projects No. MAT2014-51982-C2-R, MAT2016-80762-R, MAT2017-82970-C2-R and PID2019104150RB-I00 (including FEDER funding) and the Aragón Regional government (Project No. E26). P. J.-C. acknowledges Spanish MECD for support through FPU program (reference FPU014/02546). D.B. acknowledges support from MINECO (Spain) through an FPI program (BES-2017079688). Authors acknowledge the Advanced Microscopy Laboratory-INA University of Zaragoza for offering access to their instruments.

\section{DATA AVAILABILITY}

The data that support the findings of this study are available from the corresponding author upon reasonable request.

${ }^{1}$ G. E. W. Bauer, E. Saitoh, and B. J. van Wees, "Spin caloritronics," Nat. Mater. 11, 391-399 (2012).

${ }^{2}$ T. Kuschel and G. Reiss, "Spin orbitronics: Charges ride the spin wave," Nat. Nanotechnol. 10, 22-24 (2015).

${ }^{3}$ K. Uchida, H. Adachi, T. Ota, H. Nakayama, S. Maekawa, and E. Saitoh, "Observation of longitudinal spin-Seebeck effect in magnetic insulators," Appl. Phys. Lett. 97, 172505 (2010).

${ }^{4}$ J. Xiao, G. E. W. Bauer, E. Uchida, K.and Saitoh, and S. Maekawa, "Theory of magnon-driven spin Seebeck effect," Phys. Rev. B 81, 214418 (2010), arXiv:1009.0318.

${ }^{5}$ K. Morrison, A. J. Caruana, and C. Cox, "Towards a standard spin Seebeck measurement," arXiv, 1705.02491 (2017), arXiv:1705.02491.

${ }^{6}$ A. Sola, P. Bougiatioti, M. Kuepferling, D. Meier, G. Reiss, M. Pasquale, T. Kuschel, and V. Basso, "Longitudinal spin Seebeck coefficient: heat flux vs. temperature difference method," Sci. Rep. 7, 46752 (2017), 1701.03285. ${ }^{7}$ M. Agrawal, V. I. Vasyuchka, A. A. Serga, A. Kirihara, P. Pirro, T. Langner, M. B. Jungfleisch, A. V. Chumak, E. T. Papaioannou, and B. Hillebrands, "Role of bulk-magnon transport in the temporal evolution of the longitudinal spin-seebeck effect," Phys. Rev. B 89, 224414 (2014).

${ }^{8}$ H. Adachi, K. I. Uchida, E. Saitoh, and S. Maekawa, "Theory of the Spin Seebeck Effect,” Rep. Prog. Phys. 76, 36501 (2013).

${ }^{9}$ S. Hoffman, K. Sato, and Y. Tserkovnyak, "Landau-lifshitz theory of the longitudinal spin seebeck effect," Phy. Rev. B 88, 064408 (2013).

${ }^{10}$ S. M. Rezende, R. L. Rodríguez-Suárez, R. O. Cunha, A. R. Rodrigues, F. L. a. Machado, G. a. Fonseca Guerra, J. C. Lopez Ortiz, and A. Azevedo, "Magnon spin-current theory for the longitudinal spin-Seebeck effect," Physi. Rev. B 89, 144116 (2014).

${ }^{11}$ U. Ritzmann, D. Hinzke, and U. Nowak, "Propagation of thermally induced magnonic spin currents,” Phys. Rev. B 89, 024409 (2014).

${ }^{12}$ S. M. Rezende, R. L. Rodríguez-Suárez, R. O. Cunha, J. C. López Ortiz, and A. Azevedo, "Bulk magnon spin current theory for the longitudinal spin seebeck effect," J. Magn. Magn. Mater. 400, 171-177 (2016).

${ }^{13}$ K. Uchida, M. Ishida, T. Kikkawa, A. Kirihara, T. Murakami, and E. Saitoh, "Longitudinal spin Seebeck effect: from fundamentals to applications." J. Phys. Condens. Matter 26, 343202 (2014).

${ }^{14}$ J. Kimling, G.-M. Choi, J. T. Brangham, T. Matalla-Wagner, T. Huebner, T. Kuschel, F. Yang, and D. G. Cahill, "Picosecond spin seebeck effect," Phys. Rev. Lett. 118, 057201 (2017).

${ }^{15}$ T. S. Seifert, S. Jaiswal, J. Barker, S. T. Weber, I. Razdolski, J. Cramer, O. Gueckstock, S. F. Maehrlein, L. Nadvornik, S. Watanabe, C. Ciccarelli, A. Melnikov, G. Jakob, M. Münzenberg, S. T. B. Goennenwein, G. Woltersdorf, B. Rethfeld, P. W. Brouwer, M. Wolf, M. Kläui, and T. Kampfrath, "Femtosecond formation dynamics of the spin seebeck effect revealed by terahertz spectroscopy," Nat. Commun. 9, 2899 (2018).

${ }^{16}$ C. Y. Guo, C. H. Wan, X. Wang, C. Fang, P. Tang, W. J. Kong, M. K. Zhao, L. N. Jiang, B. S. Tao, G. Q. Yu, and X. F. Han, "Magnon valves based on yig/nio/yig all-insulating magnon junctions," Phys. Rev. B 98, 134426 (2018).

${ }^{17}$ A. Sola, M. Kuepferling, V. Basso, M. Pasquale, T. Kikkawa, K. Uchida, and E. Saitoh, "Evaluation of thermal gradients in longitudinal spin seebeck effect measurements," Journal of Applied Physics 117, 17C510 (2015), https://doi.org/10.1063/1.4916762.

${ }^{18}$ A. J. Caruana, M. D. Cropper, J. Zipfel, Z. Zhou, G. D. West, and K. Morrison, "Demonstration of polycrystalline thin film coatings on glass for spin seebeck energy harvesting," physica status solidi (RRL) - Rapid Research Letters 10, 613-617 (2016), https://onlinelibrary.wiley.com/doi/pdf/10.1002/pssr.201600128.

${ }^{19}$ G. Venkat, C. D. W. Cox, D. Voneshen, A. J. Caruana, A. Piovano, M. D. Cropper, and K. Morrison, "Magnon diffusion lengths in bulk and thin film $\mathrm{fe}_{3} \mathrm{O}_{4}$ for spin seebeck applications," Phys. Rev. Materials 4, 075402 (2020).

${ }^{20}$ R. Ramos, M. H. Aguirre, A. Anadón, J. Blasco, I. Lucas, K. Uchida, P. A. Algarabel, L. Morellón, E. Saitoh, and M. R. Ibarra, "Anomalous Nernst effect of $\mathrm{Fe}_{3} \mathrm{O}_{4}$ sinlge crystal,” Phys. Rev. B 90, 054422 (2014). 
${ }^{21}$ P. Bougiatioti, C. Klewe, D. Meier, O. Manos, O. Kuschel, J. Wollschläger, L. Bouchenoire, S. D. Brown, J.-M.-. Schmalhorst, G. Reiss, and T. Kuschel, "Quantitative Disentanglement of the Spin Seebeck , Proximity-Induced, and Ferromagnetic-Induced Anomalous Nernst Effect in Normal-Metal-Ferromagnet Bilayers," Phys. Rev. Lett. 119, 227205 (2017).

${ }^{22} \mathrm{M}$. Althammer, "Pure spin currents in magnetically ordered insulator/normal metal heterostructures." J. Phys. D: Appl. Phys. 51, 313001 (2018).

${ }^{23}$ R. Ramos, T. Kikkawa, A. Anadón, I. Lucas, T. Niizeki, K. Uchida, P. A. Algarabel, L. Morellón, M. H. Aguirre, M. R. Ibarra, and E. Saitoh, "Interface-induced anomalous nernst effect in $\mathrm{fe}_{3} \mathrm{O}_{4} / \mathrm{pt}$ based heterostructures," Applied Physics Letters 114, 113902 (2019), https://doi.org/10.1063/1.5063553.

${ }^{24}$ H. B. Vasili, M. Gamino, J. Gàzquez, F. Sánchez, M. Valvidares, P. Gargiani, E. Pellegrin, and J. Fontcuberta, "Magnetoresistance in hybrid pt/cofe2o4 bilayers controlled by competing spin accumulation and interfacial chemical reconstruction," ACS Applied Materials \& Interfaces 10, 12031-12041 (2018), pMID: 29546753, https://doi.org/10.1021/acsami.8b00384.

${ }^{25}$ P. Jiménez-Cavero, I. Lucas, A. Anadón, R. Ramos, T. Niizeki, M. H. Aguirre, P. A. Algarabel, K. Uchida, M. R. Ibarra, E. Saitoh, and L. Morellón, "Spin Seebeck effect in insulating epitaxial $\gamma-\mathrm{Fe}_{2} \mathrm{O}_{3}$ thin films," APL Mater. 5, 026103 (2017).

${ }^{26}$ A. Kehlberger, U. Ritzmann, D. Hinzke, E. J. Guo, J. Cramer, G. Jakob, M. C. Onbasli, D. H. Kim, C. A. Ross, M. B. Jungfleisch, B. Hillebrands, U. Nowak, and M. Kläui, "Length Scale of the Spin Seebeck Effect," Phys. Rev. Lett. 115, 096602 (2015).

${ }^{27}$ A. Sola, V. Basso, M. Kuepferling, M. Pasquale, D. C. né Meier, G. Reiss, T. Kuschel, T. Kikkawa, K. Uchida, E. Saitoh, H. Jin, S. J. Watzman, S. Boona, J. Heremans, M. B. Jungfleisch, W. Zhang, J. E. Pearson, A. Hoffmann, and H. W. Schumacher, "Spincaloritronic measurements: A round robin comparison of the longitudinal spin seebeck effect," IEEE Transactions on Instrumentation and Measurement 68, 1765-1773 (2019).

${ }^{28}$ T. M. Tritt, Thermal Conductivity: Theory, properties and apllications (Kluwer Academic/Plenum Publishers, New York, 2004).

${ }^{29}$ D. G. Cahill, M. Katiyar, and J. R. Abelson, "Thermal conductivity of aSi:H thin films," Phys. Rev. B 50, 6077-6081 (1994).

${ }^{30}$ S. M. Lee and D. G. Cahill, "Heat transport in thin dielectric films," J. Appl. Phys. 81, 2590-2595 (1997).
${ }^{31}$ W. Eerenstein, T. T. M. Palstra, T. Hibma, and S. Celotto, "Origin of the increased resistivity in epitaxial fe ${ }_{3} \mathrm{O}_{4}$ films," Phys. Rev. B 66, 201101 (2002).

${ }^{32}$ E. Langenberg, E. Ferreiro-Vila, V. Leborán, A. O. Fumega, V. Pardo, and F. Rivadulla, "Analysis of the temperature dependence of the thermal conductivity of insulating single crystal oxides," APL Materials 4, 104815 (2016), https://doi.org/10.1063/1.4966220.

${ }^{33} \mathrm{G}$. A. Slack, "Thermal conductivity of mgo, $\mathrm{al}_{2} \mathrm{O}_{3}, \mathrm{mgal}_{2} \mathrm{O}_{4}$, and $\mathrm{fe}_{3} \mathrm{O}_{4}$ crystals from $3^{\circ}$ to $300^{\circ} \mathrm{k}$," Phys. Rev. 126, 427-441 (1962).

${ }^{34}$ M. Schreier, A. Kamra, M. Weiler, J. Xiao, G. E. W. Bauer, R. Gross, and S. T. B. Goennenwein, "Magnon, phonon, and electron temperature profiles and the spin seebeck effect in magnetic insulator/normal metal hybrid structures," Phzs. Rev. B 88, 094410 (2013).

${ }^{35}$ R. M. Costescu, M. A. Wall, and D. G. Cahill, "Thermal conductance of epitaxial interfaces," Phys. Rev. B 67, 054302 (2003).

${ }^{36}$ A. Prakash, B. Flebus, J. Brangham, F. Yang, Y. Tserkovnyak, and J. P. Heremans, "Evidence for the role of the magnon energy relaxation length in the spin seebeck effect," Phys. Rev. B 97, 020408 (2018).

${ }^{37}$ K. Uchida, H. Adachi, T. Kikkawa, A. Kirihara, M. Ishida, S. Yorozu, S. Maekawa, and E. Saitoh, "Thermoelectric generation based on spin seebeck effects," Proceedings of the IEEE 104, 1946-1973 (2016).

${ }^{38}$ E. Saitoh, M. Ueda, H. Miyajima, and G. Tatara, "Conversion of spin current into charge current at room temperature: Inverse spin-Hall effect," Appl. Phys. Lett. 88, 1-4 (2006).

${ }^{39}$ N. Nagaosa, J. Sinova, S. Onoda, A. H. MacDonald, and N. P. Ong, "Anomalous hall effect," Rev. Mod. Phys. 82, 1539-1592 (2010).

${ }^{40}$ A. Hoffmann, "Spin Hall Effects in Metals," IEEE Trans. Magn. 49, 51725193 (2013).

${ }^{41}$ J. Sinova, S. O. Valenzuela, J. Wunderlich, C. H. Back, and T. Jungwirth, "Spin Hall effects," Rev. Mod. Phys. 87, 1213-1260 (2015).

${ }^{42}$ A. Anadón, R. Ramos, I. Lucas, P. A. Algarabel, L. Morellón, M. R. Ibarra, and M. H. Aguirre, "Characteristic length scale of the magnon accumulation in Fe3O4/Pt bilayer structures by incoherent thermal excitation," Appl. Phys. Lett. 109 (2016), 10.1063/1.4955031.

${ }^{43}$ T. Noack, H. Y. Musiienko-Shmarova, T. Langner, F. Heussner, V. Lauer, B. Heinz, D. A. Bozhko, V. I. Vasyuchka, A. Pomyalov, V. S. L'vov, B. Hillebrands, and A. A. Serga, "Spin Seebeck effect and ballistic transport of quasi-acoustic magnons in room-temperature yttrium iron garnet films,” J. Phys. D: Appl. Phys. 51, 234003 (2018). 


\title{
SUPPLEMENTARY MATERIAL FOR
}

\section{Quantification of the interfacial and bulk contributions to the longitudinal spin Seebeck effect}

\author{
P. Jiménez-Cavero, ${ }^{1,2}$ I. Lucas, ${ }^{1,2, a)}$ D. Bugallo, ${ }^{3}$ C. López-Bueno, ${ }^{3}$ R. Ramos, ${ }^{3}$ P. A. Algarabel, $, 2,1$ \\ M. R. Ibarra,,${ }^{1,2,4}$ F. Rivadulla,${ }^{3}$ and L. Morellón ${ }^{1,2}$ \\ 1) Instituto de Nanociencia y Materiales de Aragón, Universidad de Zaragoza-CSIC, 50018 Zaragoza, Spain \\ 2) Departamento de Física de la Materia Condensada, Universidad de Zaragoza, 50009 Zaragoza, Spain \\ 3) Centro de Investigación en Química Biolóxica e Materiais Moleculares (CIQUS), Universidade de \\ Santiago de Compostela, 15782 Santiago de Compostela, Spain \\ 4) Laboratorio de Microscopías Avanzadas, Universidad de Zaragoza, 50018 Zaragoza, Spain \\ a)ilucas@unizar.es
}

\section{The $3 \omega$ method}

The cross-plane thermal conductivity of the samples were measured by the $3 \omega$ method. ${ }^{1,2}$ In this method a thin metal resistor serves as a heater and a thermometer simultaneously. By applying an $\mathrm{AC}$ current at frequency $\omega$ and amplitude $\mathrm{I}_{0}$ through the resistor, a temperature oscillation at $2 \omega\left(\Delta T_{2 \omega}\right)$ is produced due to conventional Joule heating. The amplitude of this oscillation is indirectly measured through the third harmonic voltage, $\mathrm{V}_{3 \omega}$, from the formula given below (a detailed description can be found on Ref. 3,4):

$$
V_{3 \omega}=\frac{I_{0}}{2}\left(\frac{d R}{d T}\right) \Delta T_{2 \omega}
$$

The heating produced at frequency $2 \omega$, which depends on the frequency, $\omega$, of the applied ac current, is related to the thermal properties of the medium underneath the resistor. Specifically, by solving the one-dimensional heat equation, the following expression is obtained: ${ }^{5}$

$$
\Delta T_{2 \omega}=\frac{I_{0}^{2} R}{\pi l \kappa}\left[\frac{1}{2} \ln \left(\frac{D}{\left(\frac{w}{2}\right)^{2}}\right)-\frac{1}{2} \ln (2 \omega)+\eta-\frac{i \pi}{4}\right],
$$

where $I_{0}^{2} R$ is the power dissipated through the resistor ( $R$ is the electrical resistance of the metal line), $l$ is its length, $x$ and $D$ are, respectively, the thermal conductivity and thermal diffusivity of the material on top of which the resistance is placed, $w$ is the width of the resistor, and $\eta$ is a parameter that depends on the material used, $, 2,3,6,7$ Note that in order to consider the heat flow as being one-dimensional, the width of the resistor, $w$, should be much smaller than the penetration length of the thermal wave. ${ }^{5}$

When the sample underneath the resistor consists of a thin film and a substrate, the solution of the heat equation is slightly different. As long as the thickness of the film is much smaller than the width of the resistor $(t \ll w)$, which is our case, the transmission of heat through the film can be considered one-dimensional. In this scenario, the following expression is deduced (Ref. 2):

$$
\Delta T_{2 \omega}=\frac{P}{\pi l \kappa}\left[\frac{1}{2} \ln \left(\frac{D}{\left(\frac{w}{2}\right)^{2}}\right)-\frac{1}{2} \ln (2 \omega)+\eta-\frac{i \pi}{4}\right]+\frac{P}{\kappa_{\text {film }}} \frac{t}{w l}=\Delta T_{\text {substrate }}+\Delta T_{\text {film }}
$$

where the second term in the equation is the temperature increase produced by the film. At each frequency, this contribution of the film appears as an offset in comparison with the heating produced when just the substrate is present (Fig. S1) Then by subtraction of the heating produced 
in the substrate $\left(\Delta \mathrm{T}_{\text {substrate }}\right)$ to that obtained when measuring the film + substrate $\left(\Delta \mathrm{T}_{\text {film+substrate }}\right)$ the thermal conductivity of the thin film can be obtained from:

$$
\kappa_{f i l m}=\frac{P}{\Delta T_{\text {film }}} \frac{t}{w l} .
$$

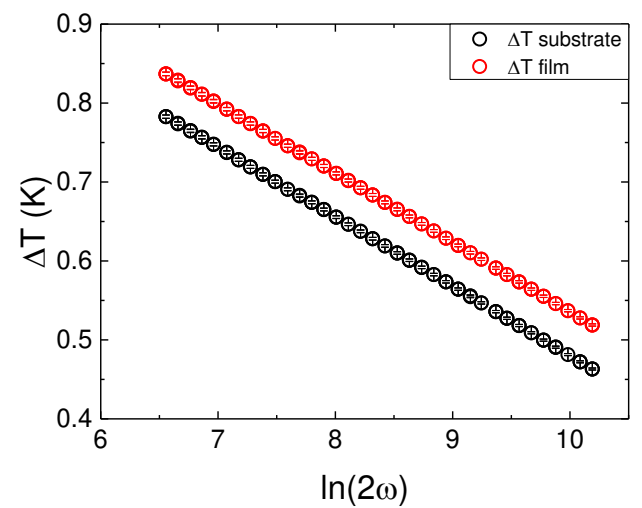

Fig S1. Temperature rise on a sample $50 \mathrm{~nm}$ thick and the $\mathrm{MgO}$ substrate.

The resistors used were made of $\mathrm{Au}$ or $\mathrm{Pt}$ (100 nm thick, $10 \mu \mathrm{m}$ width, $1 \mathrm{~mm}$ length with $10 \mathrm{~nm}$ of Cr for adhesion), by evaporation and optical lithography. The measurements were performed by applying current with a Keithley $6221 \mathrm{AC}$ source at frequencies from 56 to $2120 \mathrm{~Hz}$ to produce a heat dissipation of $30 \mathrm{~mW}$ for all samples. This samples were grown on half the substrate, leaving space on the other half to have a reference resistor on the bare substrate. The voltage drop at $3 \omega$ was measured with a Stanford Research Systems SR830 lock-in amplifier. As the ratio $\mathrm{V}_{1 \omega} / \mathrm{V}_{3 \omega}$ is around $10^{3}$, a circuit was used to cancel out the voltage at $1 \omega$, which is described in Ref. 3.

\section{Structural characterization}

X-ray characterization was performed Bruker D8 Advance high-resolution diffractometer using Cu $\mathrm{K}-\alpha 1$ radiation. $\mathrm{X}$-ray reflectivity (XRR) measurements were performed to assess the thicknesses and interfaces quality, and X-ray diffraction (XRD) patterns were recorded to ascertain the crystal quality of maghemite films.

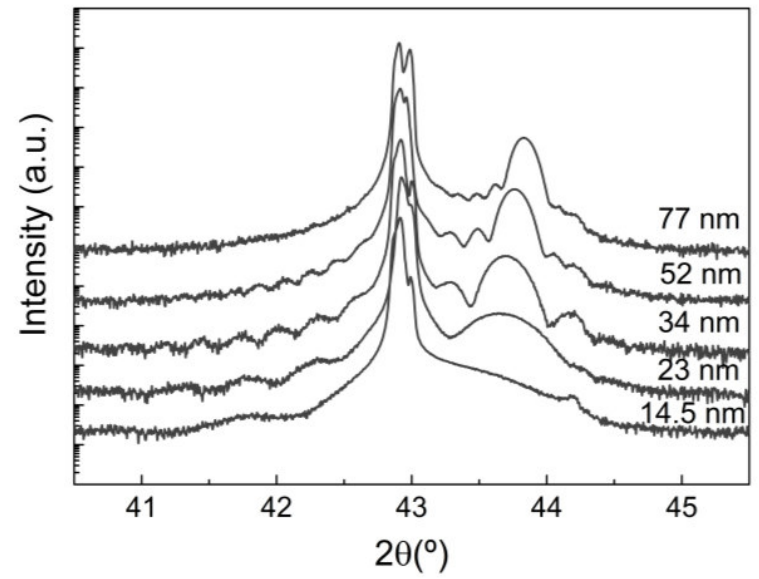

Fig S2. XRD spectra of the $\mathrm{MgO} / / \gamma-\mathrm{Fe}_{2} \mathrm{O}_{3} / \mathrm{Pt}$ samples for different $\gamma-\mathrm{Fe}_{2} \mathrm{O}_{3}$ thickness measured around the (002) $\mathrm{MgO}$ substrate Bragg peak. 
XRD patterns around the (002) MgO substrate Bragg peak are shown in Fig. S2. We observe the narrowing of the (004) maghemite peak as thickness increased, as expected from the Scherrer's formula ${ }^{8}$. Additionally, Laue oscillations are visible for all samples, indicating the existence of crystal coherence along the whole thickness of the sample.

The XRR measurements performed for every sample are shown in Fig. S3(a). The raw data are analyzed using the software Leptos from Bruker, by means of which the sample structure is simulated and fitted to the data using a simulated annealing algorithm which uses thicknesses and roughnesses as fitting parameters.

(a)

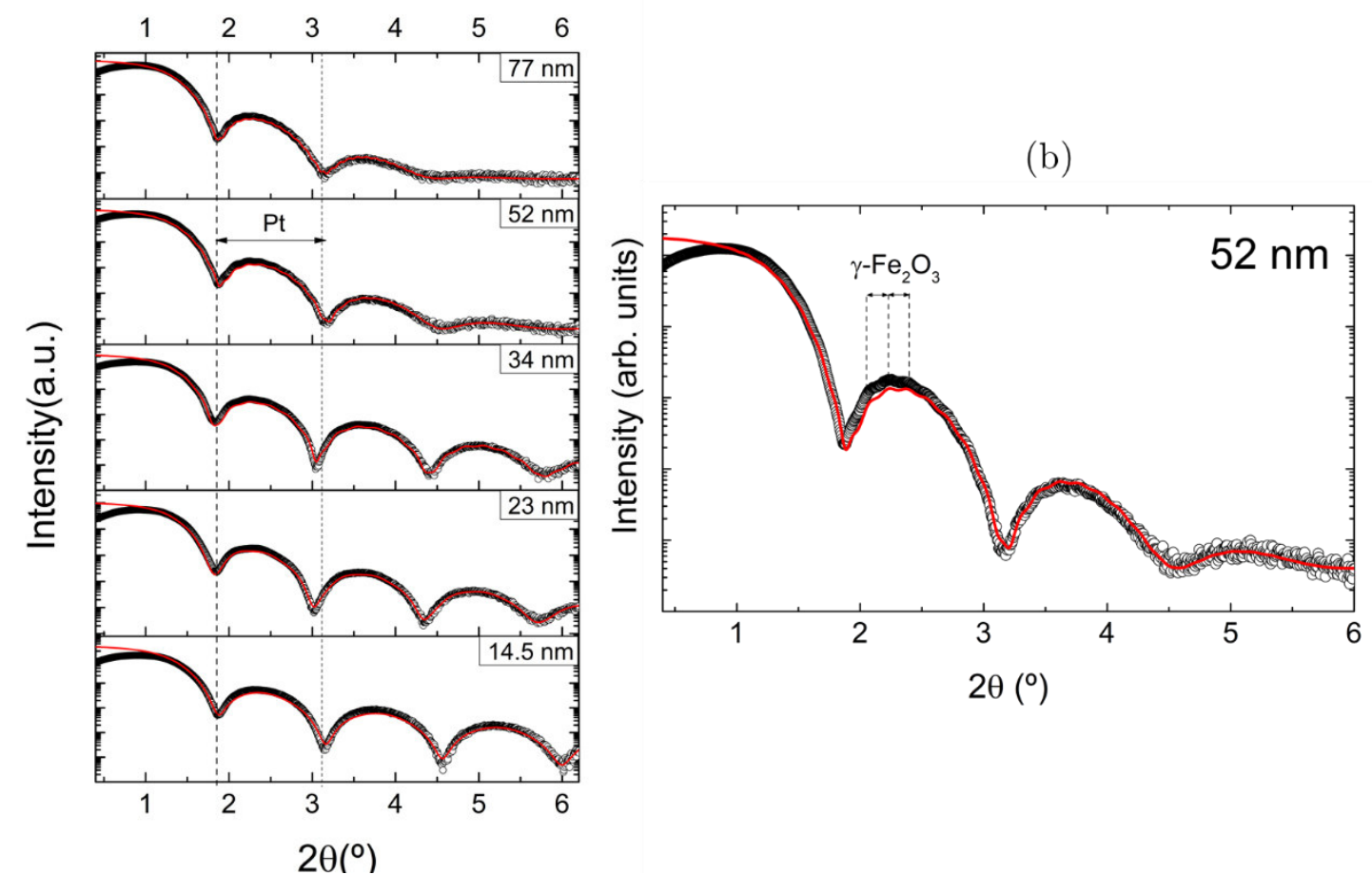

Fig S3. XRR spectra of the $\mathrm{MgO} / / \gamma-\mathrm{Fe}_{2} \mathrm{O}_{3} / \mathrm{Pt}$ samples for different $\gamma-\mathrm{Fe}_{2} \mathrm{O}_{3}$ thickness: data and fit to a simulated sample using the Leptos software.

The long-period Kiessig fringes correspond to the Pt layer and allow the extraction of its thickness, while the short-period fringes (highlighted in Fig. S3 for one of the samples) correspond to the $\gamma-\mathrm{Fe}_{2} \mathrm{O}_{3}$ layer. The obtained interface and surface roughness remains $<1 \mathrm{~nm}$ for all samples, ensuring the quality of interfaces. This was expected given that both layers $\left(\gamma-\mathrm{Fe}_{2} \mathrm{O}_{3}\right.$ and $\left.\mathrm{Pt}\right)$ were deposited in situ. As it influences the efficiency in the spin transmission across the $\gamma-\mathrm{Fe}_{2} \mathrm{O}_{3} / \mathrm{Pt}$ interface $^{9}$, interfaces with similar quality are required to enable the reliable comparison between the results obtained for all samples.

\section{Initial value of the parameters in the fit of $\Delta V_{\text {ISHE }} / \Delta T$}

The fit of Eq. 10 of the main text to the data plotted in Fig. 3 was performed using as fitting parameters $\Lambda_{\mathrm{m}}, A$, and the product $S^{\mathrm{i}} R_{\mathrm{i}}$. The respective expected order of magnitude was assigned as initial value of each parameter, as indicated in Table S1.

No lower or upper bounds to these parameters were set. 


\begin{tabular}{cc} 
Parameter & Initial value \\
\hline$\Lambda_{\mathrm{m}}$ & $10 \times 10^{-9} \mathrm{~m}$ \\
$A$ & $1 \times 10^{-9} \mathrm{~V} \mathrm{~K}^{-1}$ \\
$S^{\mathrm{i}} R_{\mathrm{i}}$ & $1 \times 10^{-9}\left(\mathrm{Vm}^{-1} \mathrm{~K}^{-1}\right)\left(\mathrm{m}^{2} \mathrm{KW}^{-1}\right)$
\end{tabular}

Table S1. Values employed for the initialization of the parameters in the fit of Eq. 10 of the main text to $\Delta V_{\mathrm{ISHE}} / \Delta T$ data.

The contact distance to measure $\Delta V_{\text {ISHE }}$ is $d_{y}=6.5 \mathrm{~mm}$. The electrical connections were made using $0.025 \mathrm{~mm} \mathrm{Al}$ wire bonded with silver paste at the sample edges. It was shown in Ref. ${ }^{10}$ that the uncontrolled size of these connections was source of experimental error responsible for data dispersion. Consequently, an error of $\pm 0.5 \mathrm{~mm}$ (half the resolution of a ruler) has been assigned to $d_{y}$ and propagated to $\Delta V_{\text {ISHE }}$ in order to address this uncertainty.

\section{References}

1. Cahill, D. G. Thermal conductivity measurement from 30 to $750 \mathrm{~K}$ : The $3 \omega$ method. Rev. Sci. Instrum. 61, 802-808 (1990).

2. Lee, S. M. \& Cahill, D. G. Heat transport in thin dielectric films. J. Appl. Phys. 81, 2590-2595 (1997).

3. López-Bueno, C., Bugallo, D., Leborán, V. \& Rivadulla, F. Sub- $\mu$ L measurements of the thermal conductivity and heat capacity of liquids. Phys. Chem. Chem. Phys. (2018) doi:10.1039/c8cp00165k.

4. Langenberg, E. et al. Analysis of the temperature dependence of the thermal conductivity of insulating single crystal oxides. APL Mater. 4, (2016).

5. Alvarez-Quintana, J. \& Rodríguez-Viejo, J. Extension of the $3 \omega$ method to measure the thermal conductivity of thin films without a reference sample. Sensors Actuators, A Phys. 142, 232-236 (2008).

6. Lee, S. M. \& Kwun, S. L. Heat capacity measurement of dielectric solids using a linear surface heater: Application to ferroelectrics. Rev. Sci. Instrum. 65, 966-970 (1994).

7. Moon, I. K., Jeong, Y. H. \& Kwun, S. I. The $3 \omega$ technique for measuring dynamic specific heat and thermal conductivity of a liquid or solid. Rev. Sci. Instrum. 67, 29-35 (1996).

8. Langford, J. I. \& Wilson, A. J. C. Scherrer after sixty years: A survey and some new results in the determination of crystallite size. J. Appl. Cryst. 11, 102-103 (1978).

9. Kehlberger, A. et al. Length Scale of the Spin Seebeck Effect. Phys. Rev. Lett. 115, 1-5 (2015).

10. Sola, A. et al. Spincaloritronic Measurements: A Round Robin Comparison of the Longitudinal Spin Seebeck Effect. IEEE Trans. Instrum. Meas. 68, 1765-1773 (2019). 